\title{
Uso do saber empírico sobre ervas condimentares para incentivo à agricultura familiar em tempo de pandemia
}

\section{RESUMO}

Saraspathy Naidoo Terroso Gama de Mendonça

naidoo@utfpr.edu.br

Universidade Tecnológica Federal do Paraná-campus Medianeira

Henry Charles Albert David Naidoo Terroso de Mendonça Brandão

henrybrandao@utfpr.edu.br Universidade Tecnológica Federal do Paraná-campus Medianeira

William Arthur Philip Louis Naidoo Terroso de Mendonça Brandão terroso@utfpr.edu.br

Universidade Tecnológica Federal do Paraná-campus Medianeira

Frieda Saicla Barros saicla@utfpr.edu.br Universidade Tecnológica Federal do Paraná-campus Curitiba

Charles Juca Busarello charles.busarello@ifpr.edu.br Universidade Tecnológica Federal do Paraná-campus Medianeira

João Paulo Vasco Avelino Joaopauloavelino1@gmail.com Universidade Tecnológica Federal do Paraná-campus Medianeira
O cenário atual demonstra que a humanidade está procurando manter a sua sobrevivência diante do quadro de pandemia Covid-19, e este momento está permeado por situações que fragilizam as populações, como por exemplo, o desemprego, fome, violência doméstica, e outras doenças crônicas como a hipertensão arterial e cardiopatias. Os dados estatísticos apontam que a população idosa tem sido mais vulnerável a doença Covid-19, porém há indícios de que adultos, jovens, crianças também são vítimas deste acometimento. $O$ artigo apresenta resultados de projeto de extensão aprovado pelo Edital PROEXT do MEC/SESu que propõe ações de empreendedorismo e de cultivo de ervas condimentares para substituir o sódio (sal de cozinha) no preparo culinário para que as famílias de agricultores e comunidades possam despertar o seu potencial de produção e criatividade como fonte de renda e de prazer e de saúde, além do exercício da solidariedade. Alia-se o cerne deste projeto ao fato de que esta doença pandêmica seja passível de atenção no nível de saúde pública devido a sua gravidade e ainda desconhecida quanto à evolução. Há que se estabelecer estratégias para implementar a tranquilidade física, mental, social e econômica das pessoas, oportunizando lhes alternativas para que possam conduzir a sua vida dentro de um foco de segurança e estabilidade econômica, emocional e de saúde física, mental e de bem-estar geral.

PALAVRAS-CHAVE: Ervas condimentares. Covid-19. Saúde. Solidariedade. Prevenção. 


\section{INTRODUÇÃO}

Desde que se declarou a pandemia, o mundo globalizado se encontra em uma situação semelhante, na qual o foco principal é a sobrevivência e a preservação da humanidade. World Health Organization (WHO) fez um manifesto recente admitindo que a doença coronavirús COVID-19, é uma ocorrência emergencial no nível de saúde pública (WHO, 2020).

O surto da doença Covid-19 foi mencionado como uma pandemia pela Organização Mundial da Saúde (WHO), e este órgão propõe que sejam desenvolvidas ações de contenção e de não disseminação do vírus (BIALEK et al., 2020). Os dados referentes a dezembro de 2019, quando surgiu a doença COVID19 na cidade de Wuhan e com uma rápida disseminação na China e quadro clínico diverso, houve a necessidade de se acompanhar a doença, mediante um estudo com 1.099 pacientes confirmados, observando-se que a média de idade era de 47 anos entre os indivíduos e $41,9 \%$ do sexo feminino (GUAN et al., 2020).

Segundo o Boletim Epidemiológico Especial do Ministério da Saúde, o número de casos confirmados por COVID-19 é de 145.328 e de 9.897 óbitos (6,8\%), estando 76.134 (52,4\%) em acompanhamento e 59.297 (40,8\%) já se recuperaram da doença até o dia 8 de maio de 2020 (BRASIL, 2020).

A distribuição dos óbitos de Síndrome Respiratória Aguda Grave (SRAG) por COVID-19 por faixa etária aponta que entre os óbitos confirmados por COVID-19, $69,0 \%$ tinham mais de 60 anos e $65,0 \%$ apresentavam pelo menos um fator de risco (BRASIL, 2020).

Há relatos na China e Itália que sugerem que a faixa etária e a presença de pelo menos uma comorbidade patogênica denotam alto risco para a evolução da doença (CHOW et al., 2020).

A realidade de um país asiático como a China, europeu como a Itália e a do Brasil, sul americano, se assemelham com respeito aos pacientes idosos acometidos pela doença COVID-19 e, diante do cenário de um testemunho global, em que um microrganismo (vírus), é causador de inúmeras mortes, e atingindo além da saúde da população, compromete também a economia das famílias, uma vez que o desemprego aumenta com a recessão motivada pelo estado de pandemia.

Tendo em vista que a doença pandêmica Covid-19 torna os indivíduos suscetíveis a sua ocorrência, principalmente idosos e pessoas que tenham comorbidades, como por exemplo cardiopatias, doenças renais, e hipertensão arterial, ações empreendedoras podem auxiliar a programar o orçamento doméstico e também a saúde física e mental.

$O$ advento da longevidade também pressupõe vários desafios. A Organização Mundial da Saúde, com o programa Saúde para Todos, no ano 2000, reconheceu a relevância da incorporação à Saúde Pública dos princípios, recursos e técnicas da Medicina Natural, por ampliar o atendimento a milhares de pessoas, atenuando seu sofrimento a custo reduzido (REVILLA, 2004), e a sua meta para reduzir a mortalidade por Doenças Crônicas Não Transmissíveis (DCNT) em 2\% ao ano demanda um enorme esforço dos países (MALACHIAS et al., 2016; MANCIA et al., 2013; NELUMBA et al., 2017).

Um dos grandes problemas de saúde pública no Brasil e no mundo é a hipertensão arterial (MION et al., 2004). As doenças cardiovasculares constituem a primeira causa de morte no Brasil, desta forma a hipertensão arterial sistêmica 
(HAS) é caracterizada como uma doença e um fator de risco e também um importante desafio em nível de saúde pública, devido a sua repercussão sobre o organismo humano (WESCHENFELDER; MARTINI, 2012).

As doenças cardiovasculares são responsáveis por mais de 250.000 mortes por ano, sendo que a hipertensão arterial sistêmica (HAS) corresponde à metade destas enfermidades (MIRANDA et al., 2002). Dentre as recomendações médicas, estão o aumento dos exercícios físicos, a redução do sal das dietas e das gorduras saturadas, o aumento de consumo de frutas e fibras, a redução do peso corporal e do consumo de álcool e tabaco, além de medidas farmacológicas (LONGO; MARTELLI; ZIMMERMANN, 2011).

Diante deste cenário grave em nível mundial e que atinge também o Brasil e os estados federativos, há que se proceder a uma reflexão sobre estratégias de ações para promover o bem-estar físico, mental, social, econômico e de saúde, num momento em que o isolamento é recomendado. Este contexto é de grande abalo econômico, comprometimento na produção de alimentos, transportes e fechamento de fronteiras entre os países afetados pela contaminação pelo vírus letal.

Este artigo teve por objetivo apresentar o resgate dos saberes populares a respeito da utilização das ervas aromáticas na alimentação. A busca do ser humano pelo prazer sensorial remonta à Antiguidade. Cita-se o caso do Rei Henrique VII, um negociante inglês astuto e muito prudente, que negou uma proposta que mais tarde viria a se tornar o investimento mais lucrativo até então conhecido, ou seja, a exploração de uma nova rota para os países de cultivo da pimenta (MENDONÇA, 2010). As taxas de comércio sobre as especiarias se elevavam em períodos bélicos, mas nem assim o consumo desaparecia (RITCHIE, 1981).

As especiarias ou condimentos conferem sabores agradáveis aos alimentos, e fazem parte da herança empírica de saberes populares, perpetuado de geração em geração. Estes saberes foram sendo absorvidos por cientistas, e transferidos para indústrias e laboratórios. Porém, ainda existem pequenos produtores, sobretudo no Estado do Paraná, que precisam ser apoiados para preservarem o plantio destas ervas, colaborarem com a preservação da biodiversidade e da saúde, e obterem renda para manterem suas famílias.

\section{METODOLOGIA}

Enfatiza-se que a presença da dimensão ética na publicação dos dados de um estudo, garante a sua integridade científica (PITHAN; VIDAL, 2017). Este estudo classifica-se quanto a sua natureza como aplicada, uma vez que se propõe a produzir conhecimentos para solução de problemas específicos (KAUARK; MANHÃES; MEDEIROS, 2010). O procedimento técnico foi o uso de um questionário fechado, construído com perguntas mais diretas, curtas, permitindo respostas previsíveis, facilitando a organização dos dados e sua análise (ARAGÃO; NETA, 2017). Segundo Ballão et al., (2012), o questionário constitui um instrumento de coleta de dados, mediante uma série de perguntas ordenadas a serem respondidas pelos informantes ou participantes. Quanto à abordagem, trata-se de uma pesquisa quantitativa e qualitativa, de forma a delimitar e mensurar o comportamento sistemático dos dados, tendo como foco a interpretação do significado do processo que os envolve (KAUARK; MANHÃES; MEDEIROS, 2010). Em relação aos objetivos, este estudo apresenta o caráter exploratório, descritivo e explicativo (GIL, 2008). 


\section{RESULTADOS E DISCUSSÕES}

O projeto de extensão aprovado pelo Edital PROEXT do MEC/SESu e encaminhado ao Comitê de Ética em Pesquisa com Seres Humanos da UTFPR, obteve o parecer consubstanciado favorável de número 678.190. Ele foi conduzido por meio de parcerias com as Secretarias de Saúde e de Ação Social do município de Medianeira-PR e com o Centro Popular de Saúde Yanten.

Foram convidadas 102 mulheres adultas e idosas, com faixa etária entre 20 e 74 anos, mediante sensibilização efetuada na Unidade Básica de Saúde dos bairros Parque Independência e Itaipu. Para o levantamento de dados iniciais, utilizou-se um questionário envolvendo aspectos relacionados às variáveis sóciodemográficas e questões abordando o conhecimento empírico a respeito das ervas aromáticas e sua utilização na alimentação.

Houve momentos de conversas em oficinas para explicar a repercussão do consumo elevado de sódio na alimentação, o cultivo e desidratação de ervas condimentares e aplicação das mesmas na elaboração de produtos alimentares e técnicas de empreendedorismo, para despertar habilidades de planejamento domiciliar para a comercialização de produtos com ervas condimentares.

Dentre os participantes, constatou-se que 25\% cursaram o Ensino Fundamental, embora, destes, somente $9 \%$ o concluíram. Aqueles que fizeram o Ensino Médio (completo ou não) correspondeu a 55\%, e 20\% cursaram, tendo ou não concluído, o Ensino Superior. Quanto à renda familiar, 79\% ganham valores correspondentes ao intervalo de um a três salários mínimos.

A Figura 1 mostra dados sobre o uso de ervas condimentares na alimentação diária. Observou-se que $71 \%$ dos participantes adiciona ervas no preparo culinário. A Figura 2 apresenta informações sobre a possibilidade de plantio próprio. Constata-se que $51 \%$ têm a prática do plantio doméstico de ervas.

Figura 1. Você e/ou sua família utiliza(m) alguma planta aromática (ervas) no preparo de refeições?

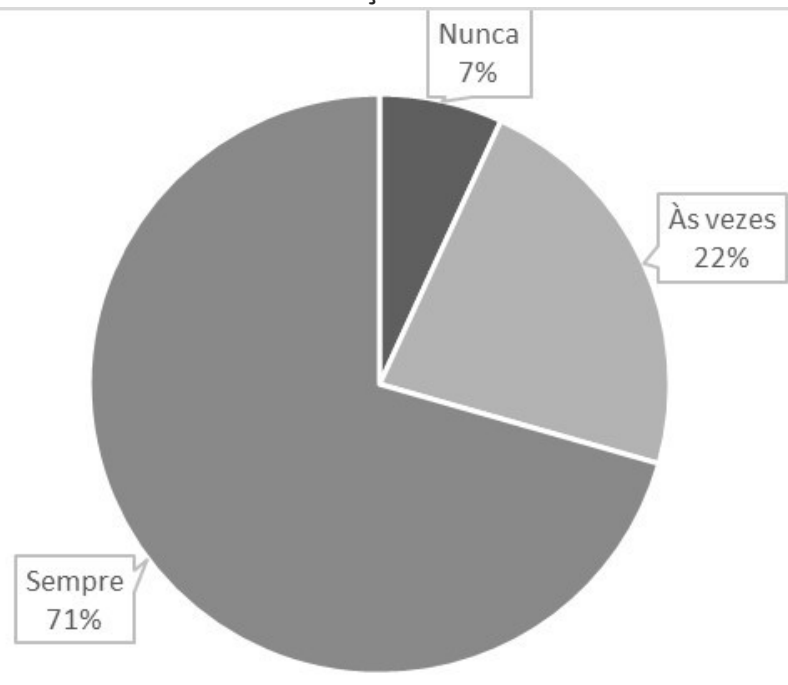

Fonte: Autoria Própria 
Figura 2. Você e/ou sua família colhe $(\mathrm{m})$ no quintal/horta as plantas aromáticas que $\operatorname{utiliza}(\mathrm{m})$ ?

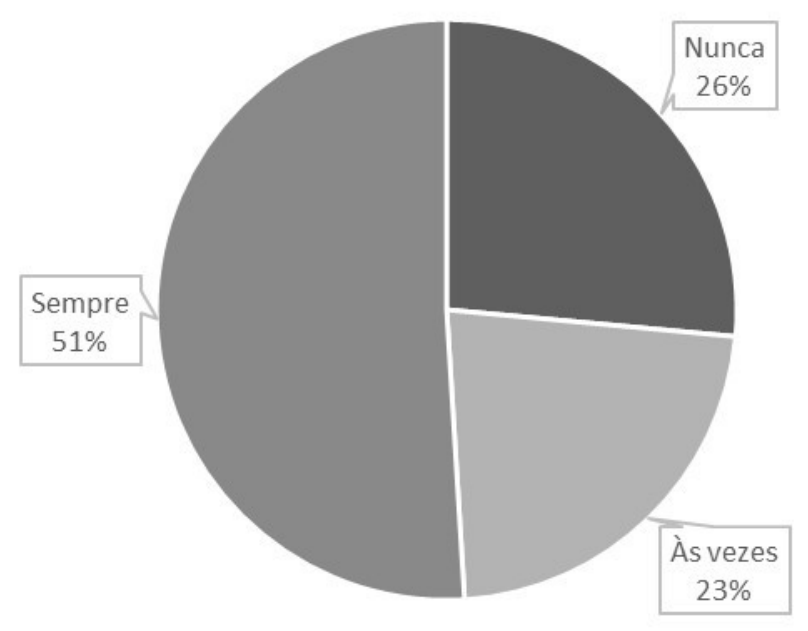

Fonte: Autoria Própria

Os resultados apresentados na Figura 3 revelam que o plantio não é um hábito frequente, pois $41 \%$ dos participantes compram as ervas condimentares e $36 \%$ as adquirem as vezes. Este comportamento pode ser alterado com as ações do Projeto de Extensão, que procura valorar a produção doméstica, e consequentemente abre possibilidades de aumentar a renda familiar.

Figura 3. Você e/ou sua família compra $(\mathrm{m})$ as plantas aromáticas que utiliza(m)?

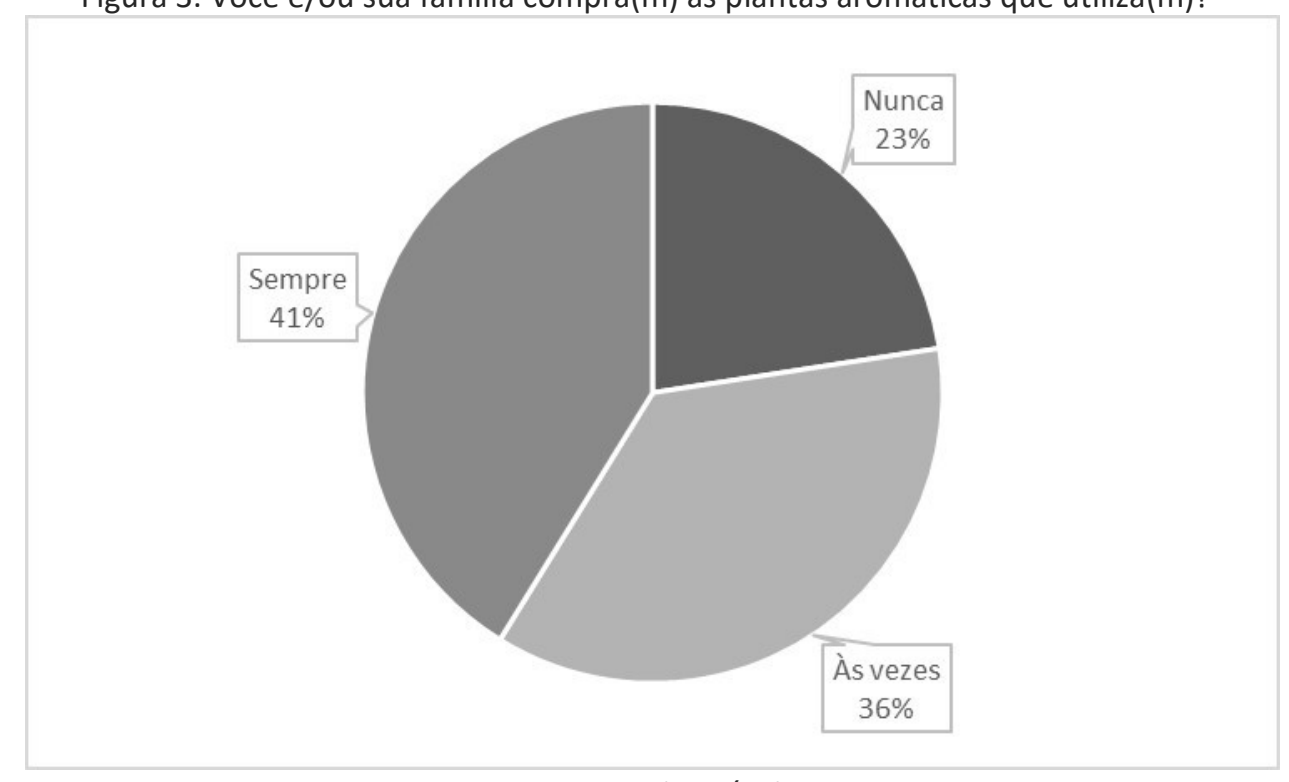

Fonte: Autoria Própria.

A Figura 4 apresenta dados sobre a obtenção das ervas com vizinhos. Verificou-se que $53 \%$ dos participantes recorrem a esta possibilidade "sempre "e "às vezes". 
Figura 4. Você e/ou sua família obtém com os vizinhos as plantas aromáticas que utiliza $(\mathrm{m})$ ?

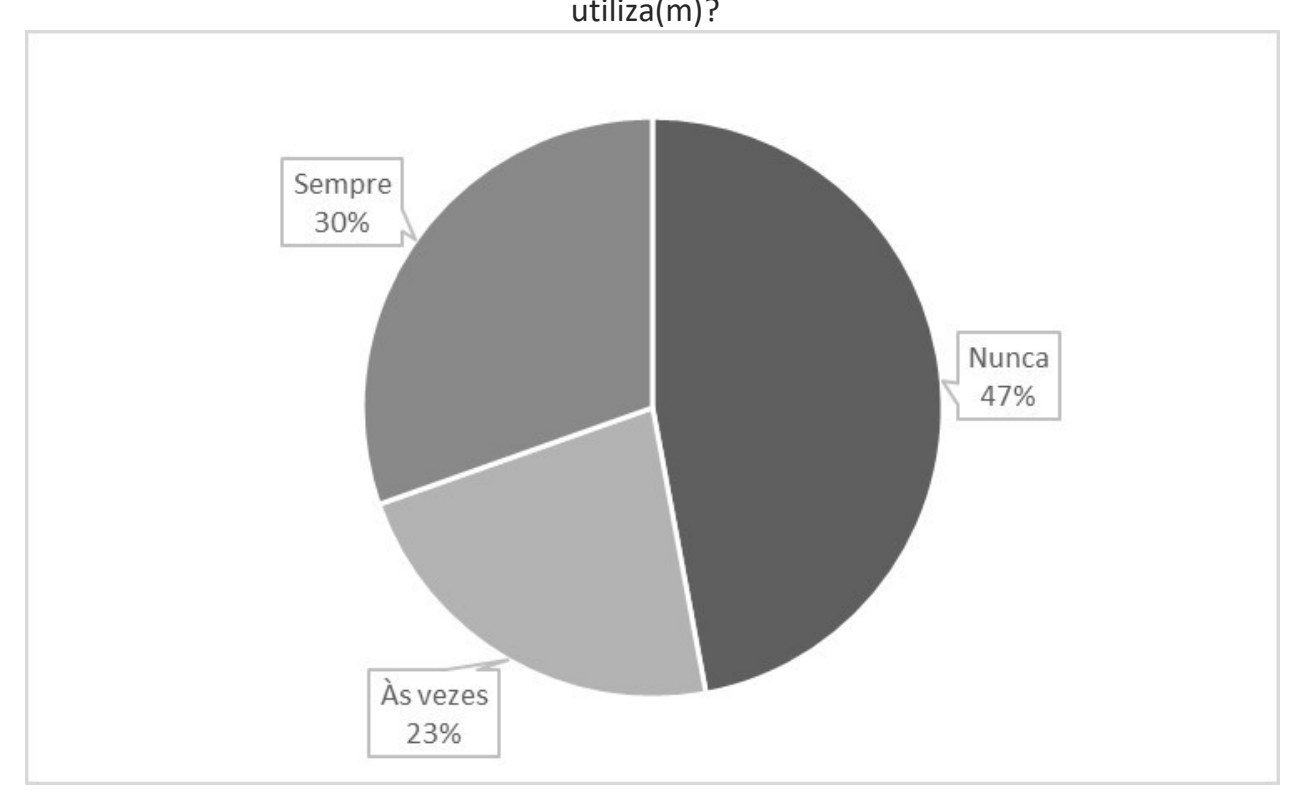

Fonte: Autoria Própria

A Figura 5 mostra que os participantes utilizam as ervas frescas na sua grande maioria (52\%). Isto sugere que as famílias podem ser preparadas para o plantio e desidratação das ervas condimentares.

Figura 5. Você e/ou sua família utiliza $(\mathrm{m})$ as folhas frescas das plantas aromáticas?

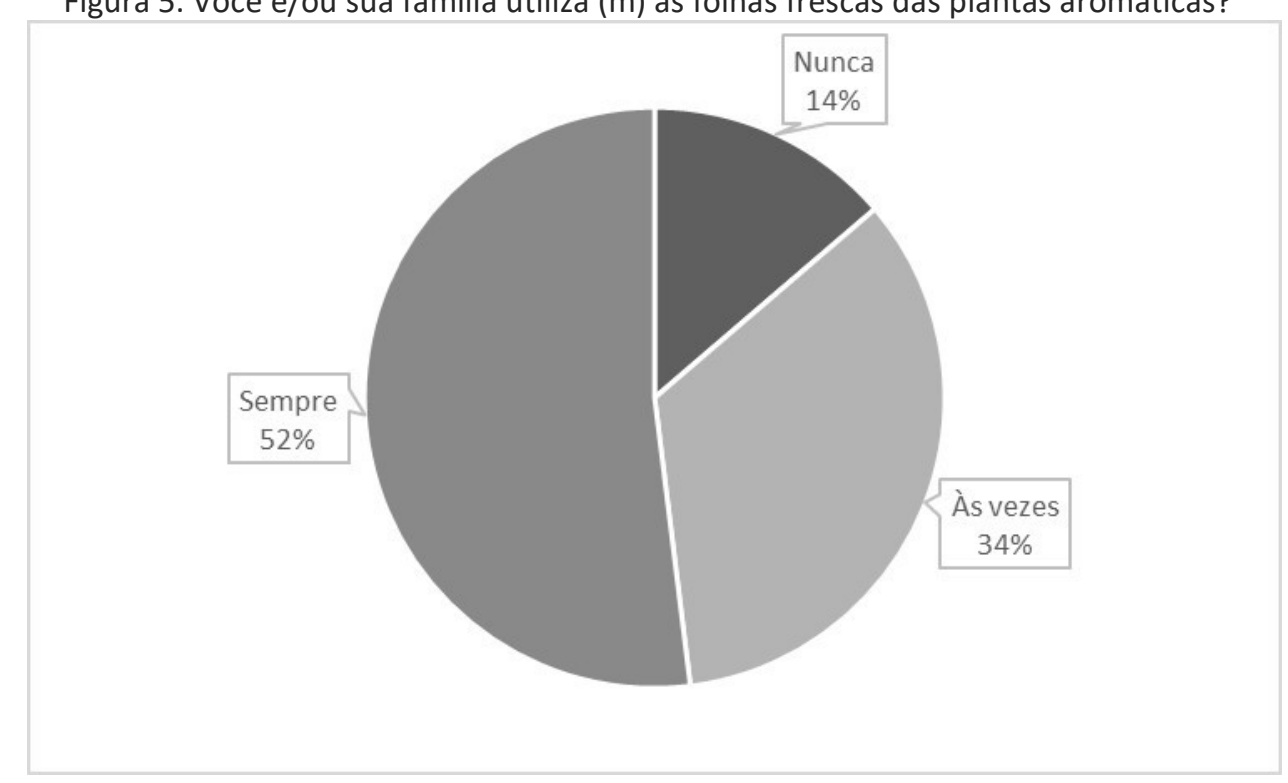

Fonte: Autoria Própria

A Figura 6 mostra que somente $35 \%$ dos participantes utilizam as folhas secas das ervas condimentares na alimentação diária. Talvez este percentual pode anunciar novas possibilidades de treinamento para a desidratação das ervas. 
Figura 6. Você e/ou sua família utiliza(m) com mais frequência as folhas secas das plantas aromáticas?

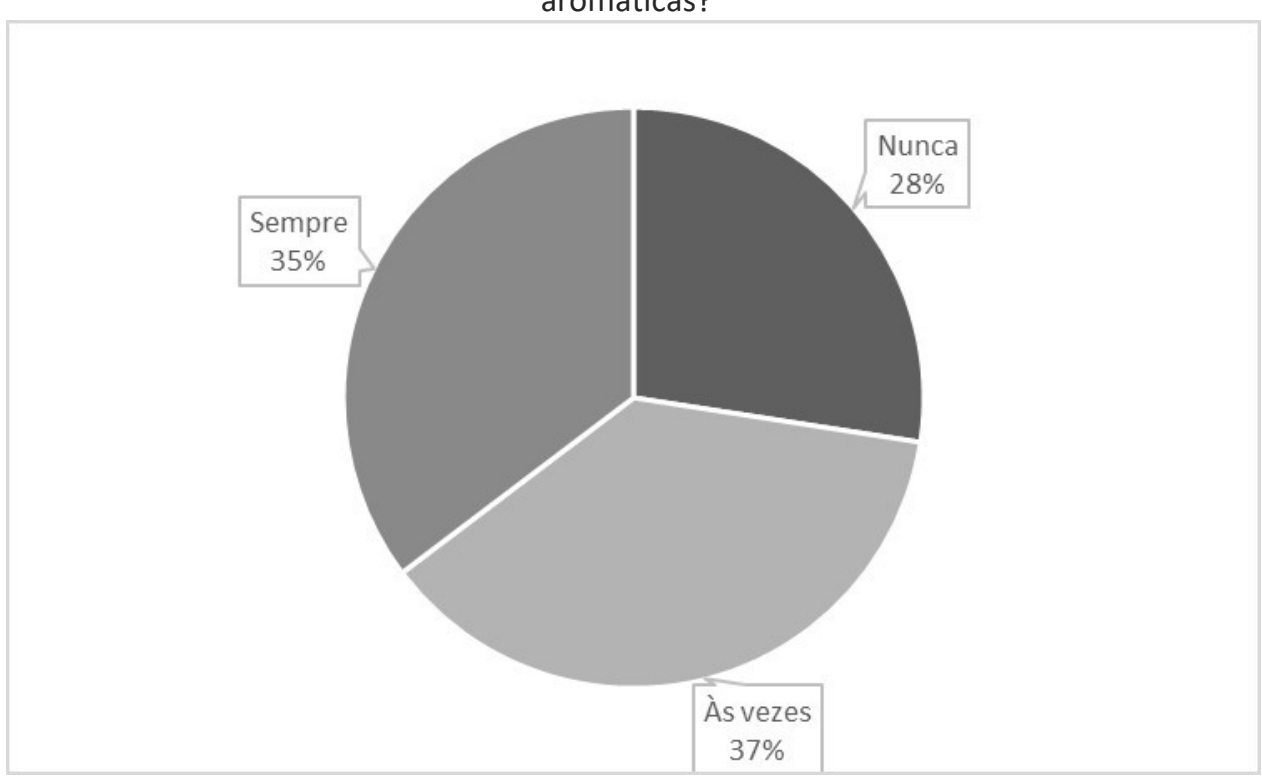

Fonte: Autoria Própria

As Figuras 7, 8, 910 e 11, ilustram as ações desenvolvidas através de Oficinas de treinamento junto aos participantes do Projeto de Extensão, sobre a prevenção da hipertensão arterial, e a substituição do sódio (sal de cozinha), por ervas condimentares.

Figura7. Palestra sobre a prevenção da hipertensão arterial e possibilidade de uso de ervas condimentares

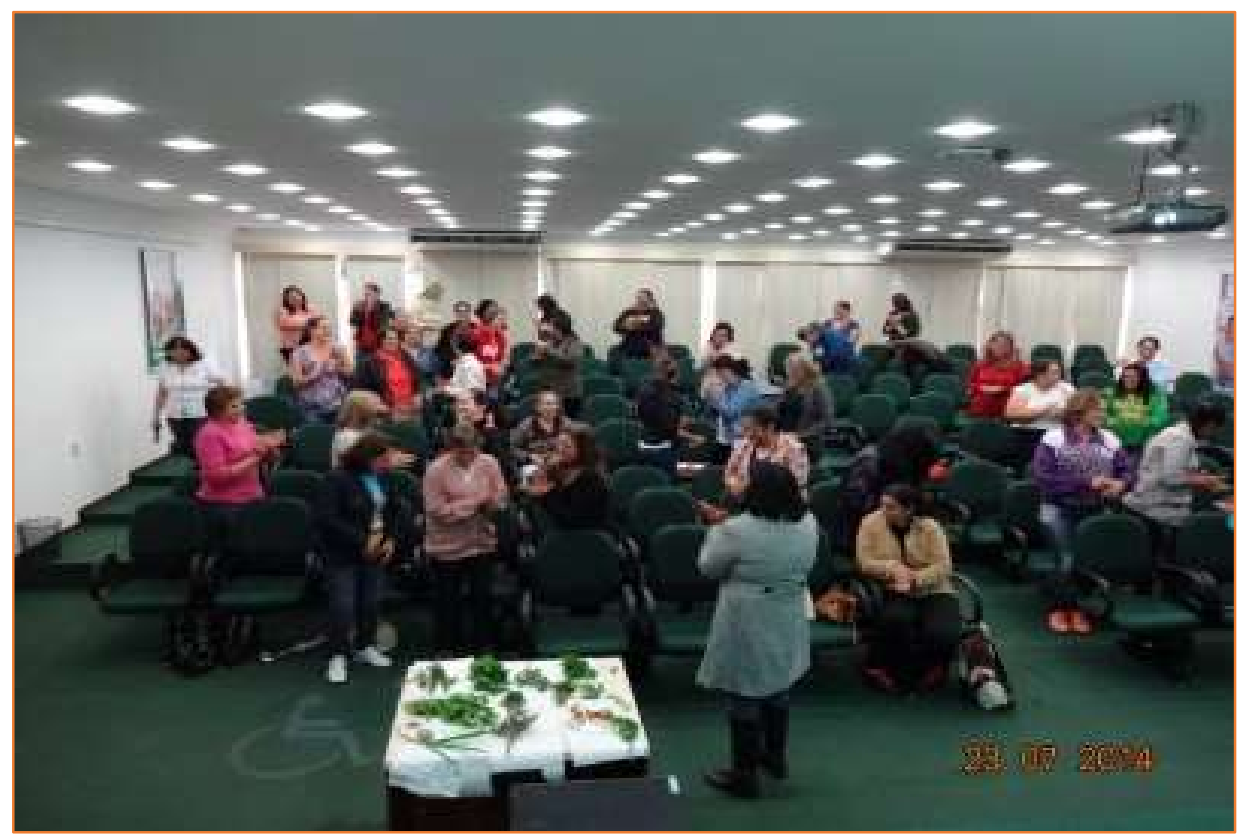

Fonte: Autoria própria 
Figura 8. Treinamento sobre o cultivo das ervas condimentares

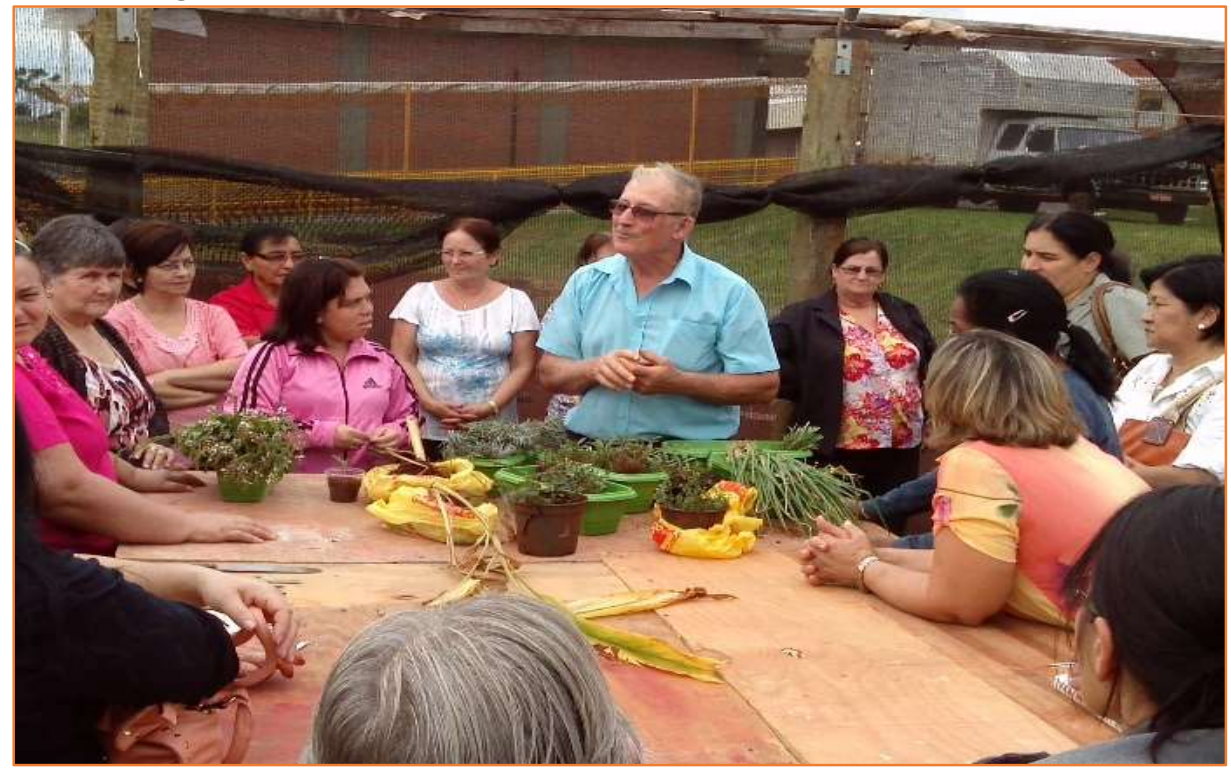

Fonte: Autoria própria

Figura 9. Desenvolvimento de iogurte com ervas condimentares

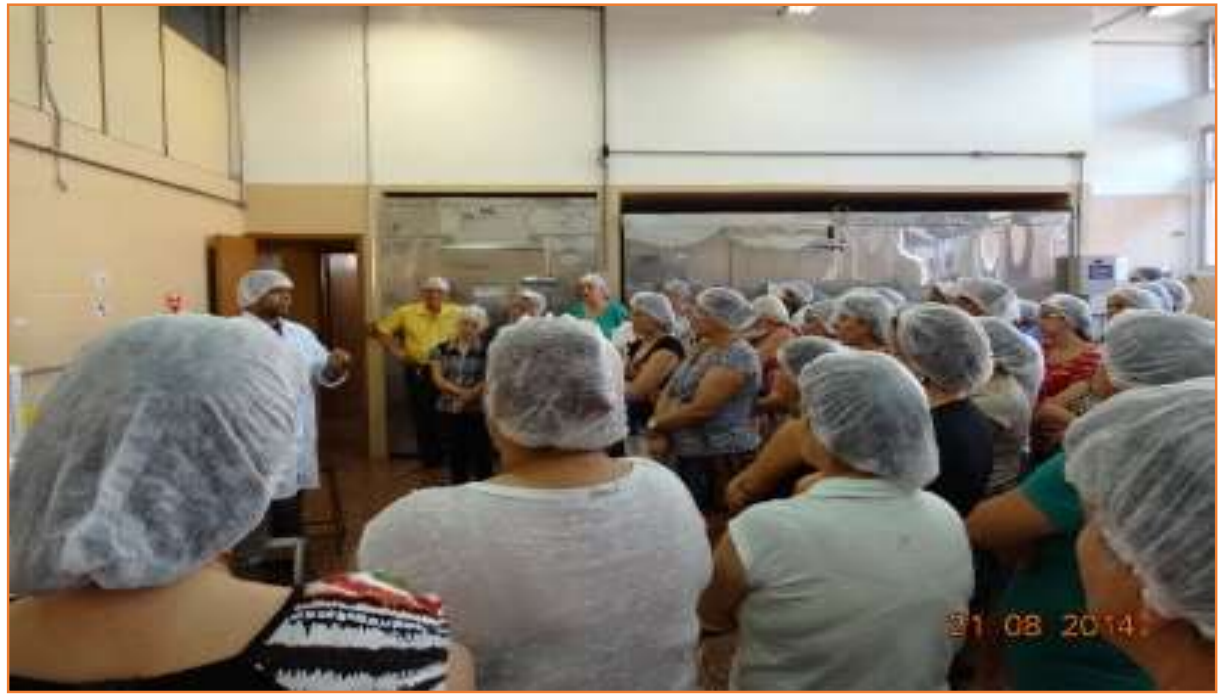

Fonte: Autoria Própria 
Figura 10. Desenvolvimento de manteiga com ervas condimentares

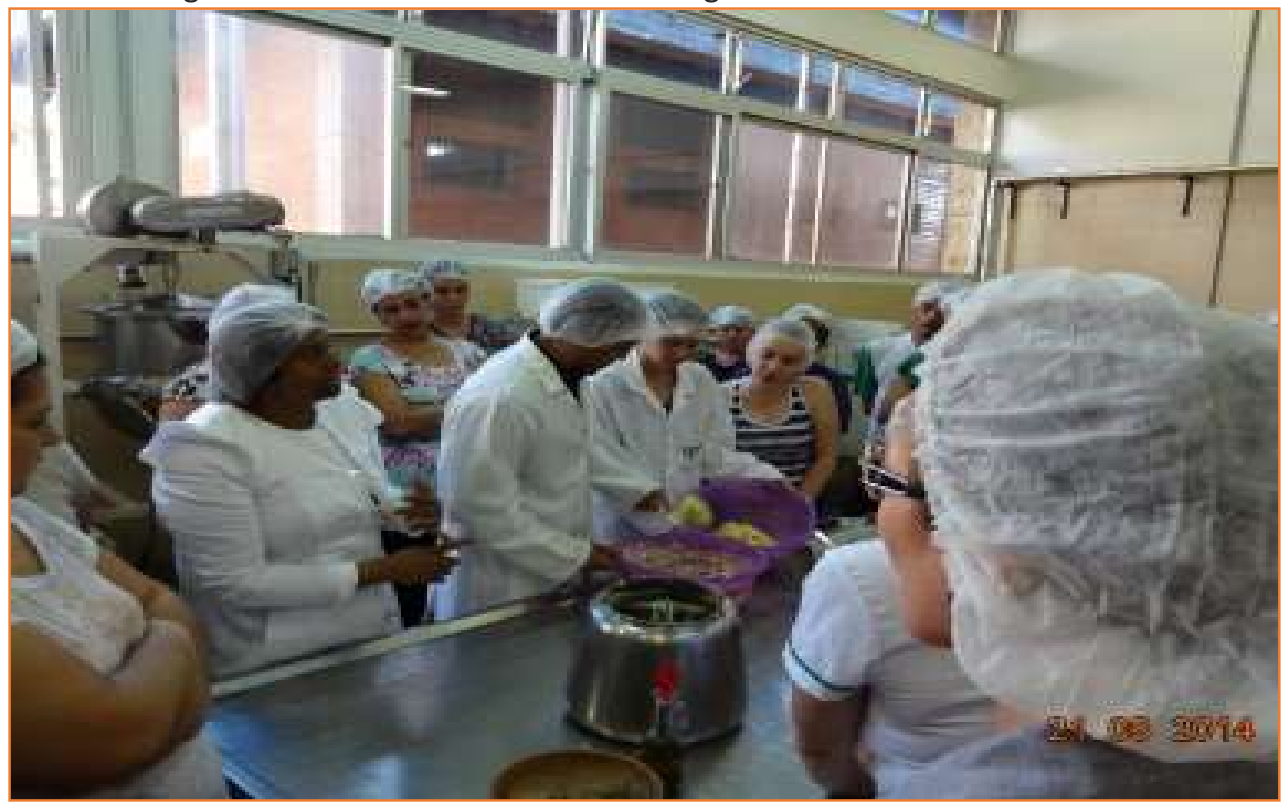

Fonte: Autoria Própria

\section{CONSIDERAÇÕES FINAIS}

Acredita-se que o Projeto de Extensão é um meio de viabilizar novas perspectivas para famílias de agricultores paranaenses em situação de vulnerabilidade à doença do coronavírus. A alternativa de consumo de ervas condimentares pode colaborar com a manutenção da saúde física e obtenção de renda e qualidade de vida sob a ótica social, física, econômica. Os resultados apresentados, por meio de questionário, apontaram para a ocorrência de hipertensão arterial entre os participantes, e o consumo de sal necessita ser reduzido. As ervas são opções saudáveis e podem alterar o sabor dos alimentos. As oficinas levantaram estas questões e introduziram saberes e conhecimentos sobre o plantio, colheita, secagem, armazenamento e aplicação das ervas aromáticas na alimentação. 


\title{
Use of empirical knowledge about condiment herbs to encourage family farming in times of pandemic
}

\begin{abstract}
The current scenario shows that humanity is trying to maintain its survival in the face of the Covid-19 pandemic, and this moment is permeated by situations that weaken populations, such as unemployment, hunger, domestic violence, and other chronic diseases. like high blood pressure, heart disease. Statistical data indicate that the elderly population has been more vulnerable to Covid-19 disease, but there are indications that adults, young people, children are also victims of this affliction. Given the fact that this pandemic disease is subject to public health attention due to its severity and still unknown as to the evolution of the disease, strategies must be established to implement people's physical, mental, social and economic well-being, providing them with alternatives so that they can lead their lives within a focus of security and economic, emotional and physical, mental health and general well-being. This project proposes actions of entrepreneurship and cultivation of condiment herbs to replace sodium (table salt) in cooking preparation so that farming families and communities can awaken their potential for production and creativity as a source of income and pleasure and health, in addition to the exercise of solidarity.
\end{abstract}

KEYWORDS: Condiment herbs. Salt. Health. Humanity. Solidarity. Prevention. 


\section{AGRADECIMENTOS}

Os pesquisadores e docentes envolvidos neste projeto agradecem a Universidade Tecnológica Federal do Paraná campus Medianeira e ao Centro Popular de Saúde Yanten e a Secretarias de Saúde e de Ação Social do município de Medianeira-Pr, pelo apoio na realização deste projeto.

\section{REFERÊNCIAS}

ARAGÃO, José Wellington Marinho.; NETA, Maria Adelina Hayne Mendes. Metodologia Científica. Salvador: UFBA, Faculdade de Educação, Superintendência de Educação a Distância, 2017. E-book (51 p). ISBN: 978-858292-131-9. Disponível

em: $<$ https://educapes.capes.gov.br/bitstream/capes/174996/2/eBook_Metodologia _CientificaEspecializacao_em_Producao_de_Midias_para_Educacao_Online_UFB A.pdf>. Acesso em: 13 maio 2020.

BALLÃO, Carmen et al. Metodologia da pesquisa. Curitiba: Instituto Federal do Paraná, 2012. (104 p). ISBN: 978-8564614-86-4. Disponível em: https://www.passeidireto.com/arquivo/2822306/metodologia-da-pesquisa. Acesso em 13 maio 2020.

BIALEK, Stephanie et al. Severe outcomes among patients with coronavirus disease 2019 (COVID-19). Centers for Disease Control and Prevention. CDC, United States, v. 69, n.12, p. 343-346, fev./mar. DOI: http://dx.doi.org/10.15585/mmwr.mm6912e2external icon 2020.Disponível em:<https://www.cdc.gov/mmwr/volumes/69/wr/mm6912e2.htm?s_cid=mm69 12e2_w\#contribAff>. Acesso em: 15 maio 2020.

BRASIL. Ministério da Saúde. Secretaria de Vigilância em Saúde. Boletim Epidemiológico Especial COE-COVID-19, no 15 | Semana Epidemiológica 19. .Brasília: Ministério da Saúde, 08 maio 2020. Disponível em: $<$ https://portalarquivos.saude.gov.br/images/pdf/2020/May/09/2020-05-06BEE15-Boletim-do-COE.pdf>. Acesso em: 16 maio 2020.

CHOW, Nancy et al. Preliminary Estimates of the Prevalence of Selected Underlying Health Conditions Among Patients with Coronavirus Disease 2019 - United States, February 12-March 28, 2020. Centers for Disease Control and Prevention, United States, v. 69, n. 13, p.382-386, fev./mar.2020. Disponível em: <https://stacks.cdc.gov/view/cdc/87230>. Acesso em: 14 maio 2020.

GIL, A. C. Métodos e Técnicas de Pesquisa Social. 6 ed. São Paulo: Atlas, 2008.

GUAN, Wei-jie et al. Clinical Characteristics of Coronavirus Disease 2019 in China. The New England Journal of Medicine, Massachusetts, v.382, n.18, p.1708-1720, 2020. DOI: 10.1056/NEJMoa2002032. Disponível em: https://www.nejm.org/doi/pdf/10.1056/NEJMoa2002032. Acesso em: 16 maio 2020. 
KAUARK, F.; MANHÃES, F.C.; MEDEIROS, C.H. Metodologia da pesquisa: guia prático. Itabuna: Via Litterarum, 2010. 88 p. Disponível em: <http://www.pgcl.uenf.br/arquivos/livrodemetodologiadapesquisa2010_011120 181549.pdf>. Acesso em: 16 maio 2020.

LONGO, M. A. T., MARTELLI, A., ZIMMERMANN, A. Hipertensão Arterial Sistêmica: aspectos clínicos e análise farmacológica no tratamento dos pacientes de um setor de Psicogeriatria do Instituto Bairral de Psiquiatria, no Município de Itapira, SP. Revista Brasileira de Geriatria e Gerontologia, Rio de Janeiro, v.14, n.2, p.:271284, 2011.DOI: https://doi.org/10.1590/S1809-98232011000200008. Disponível em: https://www.scielo.br/pdf/rbgg/v14n2/v14n2a08.pdf. Acesso em: 16 maio 2020.

MION JR, Décio et al. Sociedade Brasileira de Cardiologia. IV Diretrizes Brasileiras de Hipertensão Arterial. Arquivos Brasileiros de Cardiologia, Rio de Janeiro, v.82, n. 4, p. 1-14, 2004.Disponível em: <https://www.scielo.br/pdf/abc/v82s4/04.pdf>. Acesso em: 15 maio 2020.

MALACHIAS, Marcus Vinícius Bolivar et al. Sociedade Brasileira de Cardiologia; Sociedade Brasileira de Hipertensão; Sociedade Brasileira de Nefrologia. $7^{\mathrm{a}}$ Diretriz Brasileira de Hipertensão Arterial. Arquivos Brasileiros de Cardiologia, Rio de Janeiro, v.107, n.3, p.1-83, 2016. Disponível em: http://publicacoes.cardiol.br/2014/diretrizes/2016/05_HIPERTENSAO_ARTERIAL. pdf. Acesso em 15 maio 2020.

MANCIA, Giuseppe et al. Task Force Members. 2013 ESH/ESC Guidelines for the management of arterial hypertension: the Task Force for the management of arterial hypertension of the European Society of Hypertension (ESH) and of the European Society of Cardiology (ESC). European Heart Journal, United Kingdom, v.34, n.28, p.2159-2219, 2013. DOI: 10.1093/eurheartj/eht151. Disponível em: https://academic.oup.com/eurheartj/article/34/28/2159/451304. Acesso em: 15 maio 2020.

MENDONÇA, S. N. T.G. Nutrição. Curitiba: Editora do Livro Técnico, 2010.

MIRANDA, R.D.; PERROTTI T.C.; BELLINAZZI, V.R.; NÓBREGA, T.M.; CENDOROGLO, M.S.; TANIOLO, N. J. Hipertensão arterial no idoso: peculiaridades na fisiopatologia, no diagnóstico e no tratamento. Revista Brasileira de Hipertensão, Rio de Janeiro, v.9, n.3, p.293-300, 2002. Disponível em: http://departamentos.cardiol.br/dha/revista/9-3/hipertensaoarterial.pdf. Acesso em :15 maio 2020.

NELUMBA, J.; VIDIGAL, M.S.; FERNANDES, M.; LUANZO, L. Normas Angolanas de Hipertensão arterial - 2017. Disponível em: <http://www.angolaportal.ao/MINSA/Default.aspx>. Acesso em: 16 maio 2020.

PITHAN, Lívia Haygert.; VIDAL, Tatiane Regina Amando. O plágio acadêmico como um problema ético, jurídico e pedagógico. Revista Direito \& Justiça, Porto Alegre,

v. $39, \quad$ n. 1 , p. 77-82, jan./jun 2013. Disponível em: 
$<$ http://revistaseletronicas.pucrs.br/ojs/index.php/fadir/article/view/13676/906 6>. Acesso em 13 maio 2020.

REVILLA, J.Cultivando a saúde em hortas caseiras e medicinais. Manaus: SebraeInpa, 2004.

RITCHIE, C. I.A. Comida e Civilização. Lisboa: Assírio e Alvin, 1981.

WESCHENFELDER, M. D. ; GUE, M. J. Hipertensão arterial: principais fatores de risco modificáveis na estratégia saúde da família. Enfermería Global, Espanha, n. 26, p. 354-363, abr.2012.Disponívem em: http://scielo.isciii.es/pdf/eg/v11n26/pt_revision5.pdf. Acesso em: 13 maio 2020.

WORLD HEALTH ORGANIZATION. Coronavirus disease (COVID-19) outbreak. Disponível em: <https:// www .who .int)>. Acesso em 14 maio 2020.

Recebido: 19/05/2020

Aprovado: 16/07/2020

DOI: 10.3895/rts.v16n43.12403

Como citar: GAMA DE MENDONÇA, S.N.T.; et.al. Uso do saber empírico sobre ervas condimentares para incentivo à agricultura familiar em tempo de pandemia. R. Tecnol. Soc., Curitiba, v. 16, n. 43, p. 8-20, ed. esp.

2020. Disponível em: https://periodicos.utfpr.edu.br/rts/article/view/12403. Acesso em: XXX

Correspondência:

Direito autoral: Este artigo está licenciado sob os termos da Licença Creative Commons-Atribuição 4.0 Internacional.

\section{(c) (1)}

